\title{
Voyages avec ma patiente
}

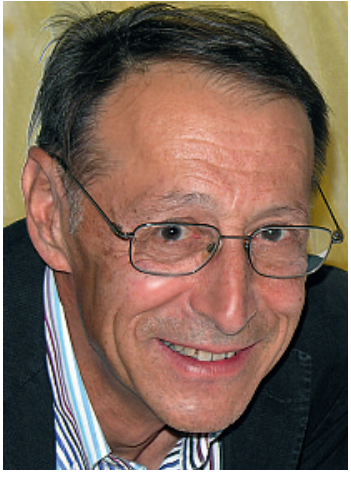

Gerhard Jenzer

Le Dr Gerhard Jenzer, Fraubrunnen est spécialiste en neurologie FMH. En collaboration avec sa patiente Sonja Balmer, il a rédigé le livre dont il est question ci-contre. Il est joignable à l'adresse gejenzer@bluewin.ch.

1 Jenzer G, Balmer S. In der Überlebensfalle. Bâle: Schwabe; 2008 www.schwabe.ch.
Ma première rencontre avec la sclérose latérale amyotrophique (SLA) lors du cours de neurologie fut effrayante. Un patient pouvant encore à peine parler en raison de sa paralysie du bulbe rachidien nous donna à ses dépens une présentation quasi insupportable de la disparition progressive et sans espoir de sa musculature. Plus tard, mon activité médicale puis celle de consilium neurologique en cas de SLA me donna l'occasion de voir des visages apeurés. J'ai ainsi rencontré Sonja Balmer, une jeune femme pleine de vie qui souffrait depuis longtemps déjà de faiblesses musculaires imprécises. La première fois, je la vis encore debout, puis en fauteuil roulant, ensuite couchée et finalement sous assistance respiratoire. Des années d'observation médicale et de découvertes mutuelles s'étaient écoulées; l'évolution de la maladie nous avait laissé plus de temps que prévu. J'étais constamment stupéfait par la manière positive sans pareille avec laquelle cette patiente affrontait son destin. Elle est restée active dans les limites du possible, est apparue dans de nombreux médias pour expliquer sa maladie, a écrit ses expériences aussi longtemps que la motricité de ses mains le lui permettait, s'est adonnée à la peinture.

Lorsque l'insuffisance respiratoire devint menaçante, Sonja Balmer eut à décider si elle voulait renoncer à la vie ou si elle allait continuer de vivre dans une dépendance totale: médicale, technique, infirmière, économique, psychologique, «palliative» ... Le laps de temps précédant sa décision définitive se caractérisa par des douleurs croissantes et le fardeau de l'issue inéluctable qui en résulterait. Où trouver le panneau indicateur, à quoi se rattacher? Elle opta pour une trachéotomie et dépend depuis lors, et pour un temps indéterminé, des fils de soie tissés par la médecine de pointe. Après que les dés ont été jetés, tout est apparu autrement que prévu. Aujourd'hui, cette patiente survit, prisonnière non seulement, physiquement, de son appareillage mais aussi du doute qui la poursuit concernant la justesse de sa décision. La qualité de vie minime atteinte dans ces circonstances, le souci de perdre sa dignité, la peur incessante de mourir plus que celle de la mort elle-même, sont continuellement soumis à des fluctuations qui l'accablent.

Il nous sembla être tout simplement de notre devoir de parcourir ce scénario dans une région quelque peu isolée et dépourvue d'accès pour rédiger un rapport de voyage, ensemble, médecin et patiente, cartographe et cliente. Nous avons estimé

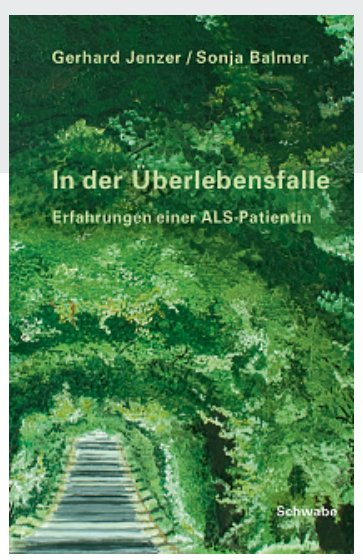
qu'il valait la peine de nous lancer dans ce projet et d'y consacrer nos efforts, eu égard à la disponibilité et plutôt à l'ordre ressenti par Sonja Balmer d'être un authentique témoin et de montrer, non seulement aux personnes concernées mais aussi à un large public, le chemin pris dans toutes ces circonstances.

Le livre «In der Überlebensfalle» [1] expose la situation médicale d'une manière compréhensible par un large public. La partie principale est toutefois constituée par le résumé des nombreuses conversations que nous avons eues avec Sonja Balmer au sujet des événements et douleurs qui frappent cette patiente SLA particulièrement au fait de son destin particulier, malgré la grande difficulté à la comprendre en raison de son aphonie. Elle estime que c'est sa tâche - importante et nécessaire - de décrire librement son vécu à l'écart de l'«objectivité» dominante, reproduite par la perspective médicale, infirmière et sociale.

Dès le début, il était clair que nous allions donner uniquement la possibilité d'observer l'option d'une assistance respiratoire continuelle lors du SLA ou d'une paralysie comparable et transmettre un point de vue, sans même essayer de donner des recommandations ou même oser un verdict. Nous avons voulu l'éviter en reconnaissant qu'un conflit intérieur ne peut être surmonté que par la personne concernée, après en avoir soupesé les avantages et les inconvénients.

Le physicien Stephen Hawking a voulu l'assistance respiratoire, la présentatrice de télévision Ulla-Carin Lindquist ou le peintre Jörg Immendorf l'ont rejetée. Ces personnalités de réputation internationale font office de guides dans la nécessaire discussion sur les soins de longue durée lors de SLA. Sonja Balmer parle d'un monde plus modeste mais qui mérite d'autant plus notre attention.

Gerhard Jenzer 